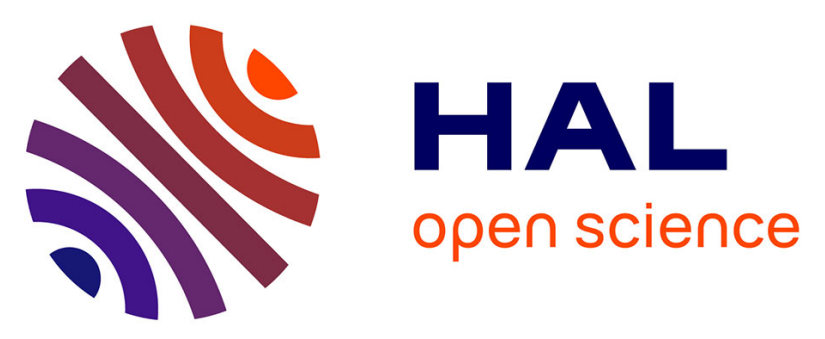

\title{
A Generalised Serre-Green-Naghdi equations for variable rectangular open channel hydraulics and its finite volume approximation
}

\author{
Mohamed Ali Debyaoui, Mehmet Ersoy
}

\section{- To cite this version:}

Mohamed Ali Debyaoui, Mehmet Ersoy. A Generalised Serre-Green-Naghdi equations for variable rectangular open channel hydraulics and its finite volume approximation. Muñoz-Ruiz M.L., Parés C., Russo G. (eds) Recent Advances in Numerical Methods for Hyperbolic PDE Systems. SEMA SIMAI Springer Series, vol 28. Springer, 2021, 978-3-030-72850-2. 10.1007/978-3-030-72850-2_11. hal-02548837v2

\author{
HAL Id: hal-02548837 \\ https://hal.science/hal-02548837v2
}

Submitted on 19 May 2020

HAL is a multi-disciplinary open access archive for the deposit and dissemination of scientific research documents, whether they are published or not. The documents may come from teaching and research institutions in France or abroad, or from public or private research centers.
L'archive ouverte pluridisciplinaire HAL, est destinée au dépôt et à la diffusion de documents scientifiques de niveau recherche, publiés ou non, émanant des établissements d'enseignement et de recherche français ou étrangers, des laboratoires publics ou privés. 


\title{
A Generalised Serre-Green-Naghdi equations for variable rectangular open channel hydraulics and its finite volume approximation
}

\author{
Mohamed Ali Debyaoui and Mehmet Ersoy
}

\begin{abstract}
We present a non-linear dispersive shallow water model which enters in the framework of sectionaveraged models. These new equations are derived up to the second order of the shallow water approximation starting from the three-dimensional incompressible and irrotational Euler system. The derivation is carried out in the case of non-uniform rectangular section and it generalises the well-known one-dimensional SerreGreen-Naghdi (SGN) equations on uneven bottom. The section-averaged model is asymptotically consistent with the Euler system in terms of mass, momentum, and energy equation which provides the richness of content for this model. We propose a well-balanced finite volume approximation and we present some numerical results to show the influence of the section variation.
\end{abstract}

Keywords. Open channel flow, Euler equations, Asymptotic approximation, Serre-Green-Naghdi equations, Free surface shallow water equations, Non-hydrostatic pressure, Dispersive model, Finite volume

\section{Introduction}

In environmental modeling of free surface flows, whenever the aspect-ratio of the domain is small enough, the shallow water approximation is introduced to obtain reduced model for which the computational cost is lower than the one implied by the numerical solution of the full three-dimensional free surface equations. One of the most widely used models to describe the channel and river motion of watercourses is the sectionaveraged free surface model [7, 2, 8] which is a generalisation of the well-known Saint-Venant system (introduced by Adhémar Jean Claude Barré de Saint-Venant in the 19th Century [18]):

$$
\begin{cases}\partial_{t} A+\partial_{x} Q & =0 \\ \partial_{t} Q+\partial_{x}\left(\frac{Q^{2}}{A}+I_{1}(x, A)\right) & =I_{2}(x, A)\end{cases}
$$

In these equations, $A=\sigma h$ is the wet area of fluid cross-section, $Q$ is the water discharge, $I_{1}(x, A)=\frac{A^{2}}{2 F_{r}^{2} \sigma}$ is the hydrostatic pressure where $F_{r}$ is the Froude's number and $I_{2}(x, A)=\frac{\sigma^{\prime}(x)}{\sigma(x)} \frac{A^{2}}{2 F_{r}{ }^{2} \sigma(x)}-\frac{A}{F_{r}{ }^{2}} d^{\prime}(x)$ is the hydrostatic pressure source term which takes into account the variation of the channel width $\sigma$ and the bottom $d$. The model (1) reduces to the well-known one-dimensional Saint-Venant equations for uniform rectangular section, i.e. if $\sigma$ is constant. The free surface model is the first order shallow water approximation

Mohamed Ali Debyaoui

Université de Toulon, IMATH EA 2134, 83957 La Garde, France, e-mail: Mohamed-Ali-Debyaoui@etud.univ-tln.fr

Mehmet Ersoy

Université de Toulon, IMATH EA 2134, 83957 La Garde, France e-mail: Mehmet.Ersoy@ univ-tln.fr 
of the section-averaged Navier-Stokes or Euler equations under suitable assumptions on the horizontal and the vertical scales (see, e.g., [11, 7, 10, 2, 8] and the reference therein).

As it is well-known, the solutions of these equations are usually suitable to approximate breaking waves with turbulent rollers for large transitions of the Froude's number. However, for small or moderate transitions, the solutions of these equations are not able to catch undular bores induced by a non-hydrostatic pressure distribution [17]. Up to our knowledge, the first section-averaged dispersive shallow water equations for quite general assumptions on the geometry of the channel was proposed in [6], thus allowing for the application of the resulting equations to natural rivers with arbitrarily shaped cross-sections. This model reads

$$
\left\{\begin{array}{l}
\partial_{t} A+\partial_{x} Q=0 \\
\partial_{t} Q+\partial_{x}\left(\frac{Q^{2}}{A}+I_{1}(x, A)+\mu_{2} D I_{1}(x, A, Q)\right)=I_{2}(x, A)+\mu_{2} D I_{2}(x, A, Q)+O\left(\mu_{2}^{2}\right)
\end{array}\right.
$$

where $D I_{1}$ and $D I_{2}$ are the non-hydrostatic counterparts of the hydrostatic pressure and the hydrostatic pressure source term. The case of non-uniform rectangular section can be regarded as the natural extension of the usual one-dimensional Serre-Green-Naghdi (SGN) equations over uneven bottom $[12,19,5]$.

In this work, we focus only on the case of a rectangular variable section. We first present the geometrical set-up in Sect. 2. Then we give the outline of the asymptotic derivation, and in particular, we show that the section-averaged model is fully consistent with the Euler system in Sect. 3. Finally, in Sect. 4, we construct a first order well-balanced finite volume approximation and we present some numerical test cases.

\section{The three-dimensional Incompressible Euler equations}

\subsection{Settings}

We consider the motion of an incompressible and irrotational fluid with constant density $\rho_{0}>0$ in a three-dimensional domain (see Fig. 1)

$$
\Omega(t)=\left\{(x, y, z) \in \mathbb{R}^{3} ; x \in\left[0, L_{c}\right], \alpha(x) \leq y-\varphi(x) \leq \beta(x), d(x) \leq z \leq \eta(t, x, y)\right\}
$$

where $\varphi$ describes the transversal variation of the channel with respect to the main channel direction, $\alpha$ and $\beta$ are the transversal limits of the channel, $L_{c}$ its length, $d$ is the bottom, $\eta$ is the free surface and $h=\eta-d$ is the water height. The boundary of the domain $\Omega(t)$ is defined by $\partial \Omega(t)$ and is decomposed into four parts: the free surface $\Gamma_{\mathrm{fs}}(t)$, the wet boundary $\Gamma_{\mathrm{wb}}(t)$, the inflow boundary $\Gamma_{\mathrm{i}}(t)$ and the outflow boundary $\Gamma_{\mathrm{o}}(t)$. The wet boundary can be decomposed itself in three parts: the bottom $\Gamma_{\mathrm{b}}(t)$, the left lateral boundary $\Gamma_{\mathrm{lb}}(t)$, and the right one $\Gamma_{\mathrm{rb}}(t)$.

Fig. 1 Geometric set-up

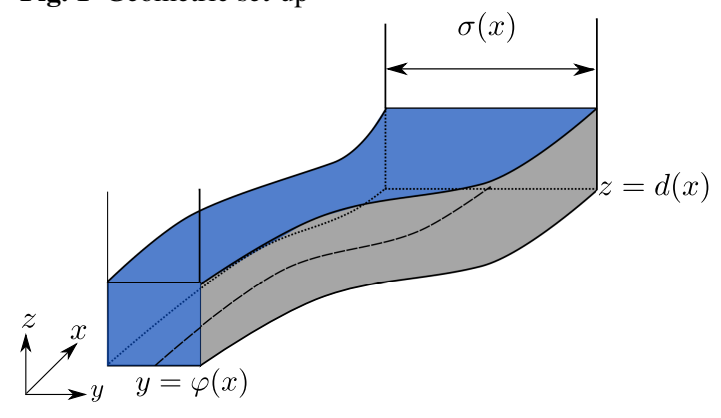


The governing equations for the motion of the fluid are the incompressible and irrotational Euler equations in $\Omega(t)$, for all $t \in(0, T]$, which can be written as follows:

$$
\begin{gathered}
\operatorname{div}[\boldsymbol{u}]=0, \\
\frac{\partial}{\partial t}(\boldsymbol{u})+\operatorname{div}[\boldsymbol{u} \otimes \boldsymbol{u}]+\nabla \frac{p}{\rho_{0}}-\boldsymbol{F}=0
\end{gathered}
$$

where $\boldsymbol{u}=(u, v, w)^{T}$ is the velocity field, $\boldsymbol{F}=(0,0,-g)^{T}$ is the gravity acceleration and $p$ is the pressure. These equations are completed by the irrotational equation:

$$
\operatorname{curl}[\boldsymbol{u}]=0 .
$$

The system is closed by suitable boundary conditions. We denote by $\boldsymbol{n}_{\mathrm{fs}}$ the outward normal to the free surface which depends on time:

$$
\boldsymbol{n}_{\mathrm{fs}}=\frac{1}{\sqrt{1+\left(\partial_{x} \eta\right)^{2}+\left(\partial_{y} \eta\right)^{2}}}\left(-\partial_{x} \eta,-\partial_{y} \eta, 1\right)^{T},
$$

and by $\boldsymbol{n}_{\mathrm{wb}}$ the outward normal to the wet boundary:

$$
\boldsymbol{n}_{\mathrm{wb}}= \begin{cases}\frac{1}{\sqrt{1+\left(\partial_{x} d\right)^{2}}}\left(\partial_{x} d, 0,-1\right)^{T} & \text { if } \boldsymbol{n}_{\mathrm{wb}}=\boldsymbol{n}_{\mathrm{b}} \\ \frac{1}{\sqrt{1+\left(\partial_{x} \alpha\right)^{2}}}\left(\partial_{x} \alpha,-1,0\right)^{T} & \text { if } \boldsymbol{n}_{\mathrm{wb}}=\boldsymbol{n}_{\mathrm{lb}} \\ \frac{1}{\sqrt{1+\left(\partial_{x} \beta\right)^{2}}}\left(\partial_{x} \beta, 1,0\right)^{T} & \text { if } \boldsymbol{n}_{\mathrm{wb}}=\boldsymbol{n}_{\mathrm{rb}}\end{cases}
$$

At the free surface, we prescribe a kinematic boundary condition

$$
\partial_{t} \eta+u \partial_{x} \eta+v \partial_{y} \eta=w \text { on } \Gamma_{\mathrm{fs}}(t)
$$

completed with the dynamical condition which takes into account the equilibrium with atmospheric stress

$$
p=p_{a} \text { on } \Gamma_{\mathrm{fs}}(t) \text {. }
$$

In the sequel, without loss of generality, we set $p_{a}=0$.

At the wet boundary, we prescribe a no-penetration condition:

$$
\begin{array}{ll}
u \partial_{x} d-w=0 & \text { on } \Gamma_{\mathrm{b}}(t), \\
u \partial_{x} \alpha-v=0 \text { on } \Gamma_{\mathrm{lb}}(t), \\
u \partial_{x} \beta+v=0 \text { on } \Gamma_{\mathrm{rb}}(t) .
\end{array}
$$

\subsection{Dimensionless Euler equations}

Let us consider the following scales involved in the wave motion: $L$ a wave-length in the longitudinal direction, $H_{2}$ a characteristic water depth, $H_{1}$ a characteristic scale of the channel width and $h_{1}$ a wavelength in the transversal direction. We then define the classical dispersive parameter $\mu_{2}$ (see e.g. [13])

$$
\mu_{2}=\frac{H_{2}^{2}}{L^{2}}
$$


and $\mu_{1}=\frac{h_{1}^{2}}{L^{2}}$ where $\mu_{1}$ is also a dispersive parameter but in the transversal direction.

In the following, we consider the asymptotic regime:

$$
h_{1}<H_{1}=H_{2} \ll L
$$

such that the following inequality holds

$$
\mu_{1}<\mu_{2}^{2} \text {. }
$$

Under these assumptions, we get the following ordering:

$$
\mu_{1}^{2}<\frac{\mu_{1}^{2}}{\mu_{2}}<\min \left(\frac{\mu_{1}^{2}}{\mu_{2}^{2}}, \mu_{1} \mu_{2}\right)<\max \left(\frac{\mu_{1}^{2}}{\mu_{2}^{2}}, \mu_{1} \mu_{2}\right)<\mu_{1}<\min \left(\frac{\mu_{1}}{\mu_{2}}, \mu_{2}^{2}\right)<\max \left(\frac{\mu_{1}}{\mu_{2}}, \mu_{2}^{2}\right)<\mu_{2} \ll 1 .
$$

We also introduce $\left(U, V=\sqrt{\mu_{1}} U, W=\sqrt{\mu_{2}} U\right)^{T}$ the scale of fluid velocity. The time scale is $T=\frac{L}{U}$. Let us define $P=\frac{p}{\rho_{0}}$ and choose the pressure scale to be $\mathcal{P}=U^{2}$.

This allows us to introduce the dimensionless quantities of time $\widetilde{t}$, space $(\widetilde{x}, \widetilde{y}, \widetilde{z})$, pressure $\widetilde{P}$, depth $\widetilde{d}$, water elevation $\widetilde{\eta}$ and velocity field $(\widetilde{u}, \widetilde{v}, \widetilde{w})$, via the following scaling relation

$$
\widetilde{x}=\frac{x}{L}, \widetilde{y}=\frac{y}{h_{1}}, \widetilde{z}=\frac{z}{H_{2}}, \widetilde{t}=\frac{t}{T}, \widetilde{P}=\frac{P}{\mathcal{P}}, \widetilde{\varphi}=\frac{\varphi}{h_{1}}, \widetilde{u}=\frac{u}{U}, \widetilde{d}=\frac{d}{H_{2}}, \widetilde{v}=\frac{v}{V}, \widetilde{\eta}=\frac{\eta}{H_{2}}, \widetilde{w}=\frac{w}{W} .
$$

Finally, we define the non-dimensional Froude's number by $F_{r}=\frac{U}{\sqrt{g H_{2}}}$.

For the sake of clarity and simplicity dropping $\widetilde{\cdot}$, using the dimensionless variables (7), and reordering the terms with respect to the powers of $\mu_{1}$ and $\mu_{2}$, the dimensionless incompressible Euler system (2) reads as follows:

$$
\begin{aligned}
\partial_{x} u+\partial_{y} v+\partial_{z} w & =0, \\
\partial_{t} u+u \partial_{x} u+v \partial_{y} u+w \partial_{z} u+\partial_{x} P & =0, \\
\mu_{1}\left(\partial_{t} v+u \partial_{x} v+v \partial_{y} v+w \partial_{z} v\right)+\partial_{y} P & =0, \\
\mu_{2}\left(\partial_{t} w+u \partial_{x} w+v \partial_{y} w+w \partial_{z} w\right)+\partial_{z} P & =-\frac{1}{F_{r}{ }^{2}} .
\end{aligned}
$$

Under this scaling, the boundary conditions (4)-(5) and (6) remain unchanged and the dimensionless irrotational equation (3) becomes

$$
\partial_{y} u=\mu_{1} \partial_{x} v, \mu_{1} \partial_{z} v=\mu_{2} \partial_{y} w, \partial_{z} u=\mu_{2} \partial_{x} w .
$$

Thanks to the ordering $\mu_{1}^{2}<\mu_{2}$ and the structure of Eqs. (12), it is natural to compute the asymptotic expansion of $u$ in two steps first with respect to $y$, then with respect to $z$. It can be achieved by first width-averaging the Euler system (8)-(11), then by depth-averaging the resulting equations. For the sake of completeness, skipping the technical details, we present the outline of the derivation. Interested readers can found the details in [6]. 
Title Suppressed Due to Excessive Length

\section{Derivation of the section-averaged model}

\subsection{Width-averaged equations}

By integrating for $s \in[\alpha(x), y]$, the first two equations of the irrotational equations (12) and the divergence equation (8), keeping in mind the boundary conditions (4)-(5) and (6), we get the following asymptotic expansions:

$$
\begin{gathered}
u(t, x, y, z)=u_{\alpha}(t, x, z)-\frac{\mu_{1}}{2} \partial_{x} \operatorname{div}_{x, z}\left[w_{\alpha}(t, x, z)(y-\alpha(x))^{2}\right]+O\left(\frac{\mu_{1}^{2}}{\mu_{2}}\right), \\
v(t, x, y, z)=-\operatorname{div}_{x, z}\left[w_{\alpha}(t, x, z)(y-\alpha(x))\right]+O\left(\frac{\mu_{1}}{\mu_{2}}\right)
\end{gathered}
$$

and

$$
w(t, x, y, z)=w_{\alpha}(t, x, z)-\frac{\mu_{1}}{2 \mu_{2}} \partial_{z} \operatorname{div}_{x, z}\left[w_{\alpha}(t, x, z)(y-\alpha(x))^{2}\right]+O\left(\frac{\mu_{1}^{2}}{\mu_{2}^{2}}\right)
$$

where $X_{\alpha}(t, x, z):=X(t, x, \alpha(x), z)$.

For a given function $(t, x, y, z) \mapsto X(t, x, y, z)$, we define its width-average by

$$
\langle X\rangle(t, x, z):=\frac{1}{\sigma(x)} \int_{\alpha(x)}^{\beta(x)} X(t, x, y, z) d y
$$

where $\sigma(x)=\beta(x)-\alpha(x)$ is the width of the channel.

Integrating Eqs. (8)-(11) for $y \in[\alpha(x), \beta(x)]$, using Leibniz integral rule, keeping in mind the boundary conditions (4)-(5) and (6), using the asymptotic expansions (13)-(15), we obtain the width-averaged Euler system:

$$
\begin{array}{ll}
\operatorname{div}_{x, z}\left[\sigma \boldsymbol{w}_{\alpha}\right] & =O\left(\frac{\mu_{1}}{\mu_{2}}\right), \\
\frac{\partial}{\partial t}\left(\sigma u_{\alpha}\right)+\operatorname{div}_{x, z}\left[\sigma u_{\alpha} \boldsymbol{w}_{\alpha}\right]+\frac{\partial}{\partial x}\left(\sigma P_{\alpha}\right)+ & =P_{\alpha} \frac{\partial \sigma}{\partial x}+O\left(\frac{\mu_{1}}{\mu_{2}}\right), \\
\mu_{2}\left(\frac{\partial}{\partial t}\left(\sigma w_{\alpha}\right)+\operatorname{div}_{x, z}\left[\sigma w_{\alpha} \boldsymbol{w}_{\alpha}\right]\right)+\frac{\partial}{\partial z}\left(\sigma P_{\alpha}\right) & =-\frac{\sigma}{{F_{r}}^{2}}+P_{\alpha} \frac{\partial \sigma}{\partial z}+O\left(\mu_{1}\right)
\end{array}
$$

where $P_{\alpha}(t, x, z)+O\left(\mu_{1}\right)=P(t, x, y, z)$ thanks to Eq. (10). The motion of the fluid is now in a two-dimensional domain:

$$
\langle\Omega\rangle(t)=\left\{(x, z) \in \mathbb{R} ; d(x) \leq z \leq \eta^{*}(t, x)\right\} .
$$

The irrotational condition (12) reduces to

$$
\frac{\partial u_{\alpha}}{\partial z}=\mu_{2} \frac{\partial w_{\alpha}}{\partial x}+O\left(\mu_{1}\right)
$$

and the boundary conditions to

$$
\begin{array}{r}
\frac{\partial \eta^{*}}{\partial t}+u_{\alpha} \frac{\partial \eta^{*}}{\partial x}=w_{\alpha}+O\left(\frac{\mu_{1}}{\mu_{2}}\right) \text { and } P_{\alpha}=O\left(\mu_{1}\right) \text { on }\left\langle\Gamma_{\mathrm{fs}}\right\rangle(t), \\
u_{\alpha} \partial_{x} d=w_{\alpha}+O\left(\frac{\mu_{1}}{\mu_{2}}\right) \text { on }\left\langle\Gamma_{\mathrm{b}}\right\rangle(t)
\end{array}
$$

where $\left\langle\Gamma_{\mathrm{fs}}\right\rangle(t)$ is the free surface boundary and $\left\langle\Gamma_{\mathrm{b}}\right\rangle(t)$ the bottom boundary of the width-averaged fluid domain $\langle\Omega\rangle(t)$. 
The function $\eta^{*}$ in the above expression depends only on $t$ and $x$. Indeed, integrating Eq. (11) for $s \in[z, \eta(t, x, y)]$, using the previous asymptotic expansions, and noting $\frac{D}{D t} w=\partial_{t} w+u \partial_{x} w+v \partial_{y} w+w \partial_{z} w$, we can write

$$
P_{\alpha}(t, x, z)=\frac{\eta(t, x, y)-z}{F_{r}^{2}}+\mu_{2} \int_{z}^{\eta(t, x, y)} \frac{D}{D t} w_{\alpha}(t, x, s) d s+O\left(\mu_{1}\right) .
$$

Thus, taking the $y$-derivative of the above expression provides

$$
0=\partial_{y} \eta\left(\frac{1}{F_{r}^{2}}+\mu_{2} \frac{D}{D t} w_{\alpha \mid z=\eta}\right)+O\left(\mu_{1}\right)=-\partial_{y} \eta \partial_{z} P_{\mid z=\eta}+O\left(\mu_{1}\right)
$$

Consequently, since $\partial_{z} P_{\mid z=\eta} \neq 0$, we get $\partial_{y} \eta=O\left(\mu_{1}\right)$. This is the so-called flat free surface approximation. Therefore, one can write

$$
\eta(t, x, y)=\eta^{*}(t, x)+O\left(\mu_{1}\right)
$$

where the * is dropped in the following.

\subsection{Depth-averaged equations}

Integrating Eq. (17) together with the first equation of System (16) for $s \in[d(x), z]$, keeping in mind Eqs. (18)-(19), we obtain

$$
u_{\alpha}(t, x, z)=u_{d}(t, x)-\mu_{2} \int_{d(x)}^{z} \partial_{x} \mathcal{S}\left(u_{d}, x, s\right) d s+O\left(\mu_{2}^{2}\right)
$$

and

$$
w_{\alpha}(t, x, z)=-\frac{1}{\sigma(x)} \frac{\partial}{\partial x}\left(u_{d}(t, x) S(x, z)\right)+O\left(\mu_{2}\right)
$$

where $\mathcal{S}(u, x, z)=\frac{1}{\sigma(x)} \frac{\partial}{\partial x}(u S(x, z)), S(x, z)=\sigma(x)(z-d(x))$ and $X_{d}(t, x)=X_{\alpha}(t, x, d(x))$.

Thanks to the flat free surface approximation (20), one can write the section-average of the velocity $u$ as follows:

$$
\bar{u}=\frac{1}{A} \int_{d(x)}^{\eta(t, x)} \int_{\alpha(x)}^{\beta(x)} u(t, x, y, z) d y d z
$$

where $A=\int_{d(x)}^{\eta(t, x)} \int_{\alpha(x)}^{\beta(x)} d y d z=\sigma(x) h(t, x)$ is the wet area, $\sigma=\beta-\alpha$ is the width of the channel and $h=\eta-d$ is the water height.

Thus, since $u(t, x, y, z)=u_{\alpha}(t, x, z)+O\left(\mu_{1}\right)=u_{d}(t, x)-\mu_{2} \int_{d(x)}^{z} \partial_{x} \mathcal{S}\left(u_{d}, x, s\right) d s+O\left(\mu_{2}^{2}\right)$, we deduce the following asymptotic expansion of $u$ :

$$
u=\bar{u}(t, x)+\mu_{2} B_{0}(\bar{u}, x, z)+O\left(\mu_{2}^{2}\right)
$$

where

$$
B_{0}(\bar{u}, x, z)=\frac{1}{A(t, x)} \int_{d(x)}^{\eta(t, x)}\left(\sigma(x) \int_{d(x)}^{z} \partial_{x} \mathcal{S}(\bar{u}, x, s) d s\right) d z-\int_{d(x)}^{z} \partial_{x} \mathcal{S}(\bar{u}, x, s) d s .
$$

Similarly, we get for $w$ :

$$
w(t, x, y, z)=-\mathcal{S}(\bar{u}, x, z)+O\left(\frac{\mu_{1}}{\mu_{2}}\right) .
$$

Using the asymptotic expansion of $u(21)$ and $w(22)$, we obtain the asymptotic expansion of the pressure $P$ at order $O\left(\mu_{2}^{2}\right)$ 


$$
P(t, x, y, z)=P_{\alpha}(t, x, z)+O\left(\mu_{1}\right)=P_{\mathrm{h}}(t, x, z)+\mu_{2} P_{\mathrm{nh}}(t, x, z)+O\left(\mu_{2}^{2}\right)
$$

where

$$
P_{\mathrm{h}}(t, x, z)=\frac{(\eta(t, x)-z)}{F_{r}^{2}}
$$

is the usual hydrostatic pressure and

$$
\begin{aligned}
P_{\mathrm{nh}}(t, x, z)= & \int_{z}^{\eta(t, x)} \frac{1}{2 \sigma(x)^{2}} \partial_{z}\left((\sigma(x) \mathcal{S}(\bar{u}, x, s))^{2}\right) d s \\
& -\int_{z}^{\eta(t, x)} \partial_{t} \mathcal{S}(\bar{u}, x, s)+\frac{\bar{u}(t, x)}{\sigma(x)} \partial_{x}(\sigma(x) \mathcal{S}(\bar{u}, x, s)) d s
\end{aligned}
$$

is the non-hydrostatic part of the pressure.

\subsection{Section-averaged model}

To end the asymptotic derivation, we integrate vertically the set of equations (16) between $d$ and $\eta$ and drop all terms of order lower than $\mu_{2}$. We get the generalised Serre-Green-Naghdi equations for non-uniform rectangular section:

$$
\left\{\begin{array}{l}
\partial_{t} A+\partial_{x} Q=0 \\
\partial_{t} Q+\partial_{x}\left(\frac{Q^{2}}{A}+I_{1}(x, A)+\mu_{2} D I_{1}(x, A, Q)\right)=I_{2}(x, A)+\mu_{2} D I_{2}+O\left(\mu_{2}^{2}\right)
\end{array}\right.
$$

where $A=\sigma h$ is the wet area, $Q$ is the water discharge, $I_{1}(x, A)=\frac{A^{2}}{2 F_{r}^{2} \sigma(x)}$ is the hydrostatic pressure, $I_{2}(x, A)=\frac{\sigma^{\prime}(x)}{\sigma(x)} \frac{A^{2}}{2 F_{r}{ }^{2} \sigma(x)}-\frac{A}{F_{r}{ }^{2}} d^{\prime}(x)$ is the hydrostatic pressure source term, $D I_{1}=\int_{d(x)}^{\eta(t, x)} P_{\mathrm{nh}}(t, x, z) \sigma(x) d z$ is the non-hydrostatic pressure and $D I_{2}=\int_{d(x)}^{\eta(t, x)} P_{\mathrm{nh}}(t, x, z) \sigma^{\prime}(x) d z-\sigma(x) P_{\mathrm{nh}}(t, x, d(x)) d^{\prime}(x)$ is the nonhydrostatic pressure source term.

Moreover, Eqs. (23) are by construction asymptotically consistent with the Euler system (8)-(11). We have the following result:

Theorem 1 System (23) admits a total energy

$$
E=A \frac{\bar{u}^{2}}{2}+A \frac{\eta}{F_{r}^{2}}-I_{1}+\frac{\mu_{2}}{2} \int_{\Omega} \mathcal{S}^{2}(\bar{u}, x, z) d y d z
$$

which satisfies the following energy equation

$$
\partial_{t} E+\partial_{x}\left(\left(E+I_{1}+\mu_{2} D I_{1}\right) \bar{u}\right)=0 .
$$

Moreover, the quantity $E$ is consistent with the total energy $\mathcal{E}=\frac{u^{2}+\mu_{1} v^{2}+\mu_{2} w^{2}}{2}+\frac{z}{F_{r}^{2}}$ of the Euler equation (8)-(11), in the sense that

$$
\partial_{t} \int_{\Omega} \mathcal{E} d y d z+\partial_{x} \int_{\Omega}(\mathcal{E}+P) u d y d z=\partial_{t} E+\partial_{x}\left(\left(E+I_{1}+\mu_{2} D I_{1}\right) \bar{u}\right)+O\left(\mu_{2}^{2}\right) .
$$

Remark 1 This is a positive feature of the approximate model (23), which provides the richness of content for this model and can be used in the estimation of the accuracy of numerical algorithms. Moreover, it is well-known that the energy conservation law plays a fundamental role in the justification of the theory of shallow water equations. 
Remark 2 As a direct consequence of (24) and (25), we are able to recover the energy conservation law of the usual models in the case of $\sigma \equiv 1$, i.e. $A=h$ :

- if $\mu_{2}=0$, we recover the classical total energy of the Saint-Venant system, namely

$$
E=\frac{h \bar{u}^{2}}{2}+\frac{h(h+2 d)}{2 F_{r}^{2}} .
$$

- if $\mu_{2} \neq 0$, we recover the classical total energy of the Serre-Green-Naghdi system (see for instance [9]), namely

$$
E=\frac{h \bar{u}^{2}}{2}+\frac{h(h+2 d)}{2 F_{r}^{2}}+\mu_{2}\left(\frac{h^{3}}{6}\left(\partial_{x} \bar{u}\right)^{2}-d^{\prime} \frac{h^{2}}{2} \partial_{x} \bar{u}+\frac{\left(d^{\prime}\right)^{2}}{2}\right) .
$$

\section{A well-balanced finite volume approximation}

The main drawback of Eqs. (23) is that it has third order terms in space which may lead to instabilities at the numerical level. Therefore, we first propose a more stable formulation of Eqs. (23) before presenting its numerical approximation.

Skipping the technical details, defining a linear operator $\mathbb{L}$ (where $\mathcal{L}$ is defined below)

$$
\mathbb{L}[A, d, \sigma](u)=A \mathcal{L}[A, d, \sigma]\left(\frac{u}{A}\right),
$$

one can show that System (23) can be written:

$$
\left\{\begin{array}{l}
\partial_{t} A+\partial_{x} Q=0 \\
\left(I_{d}-\mu_{2} \mathbb{L}[A, d, \sigma]\right)\left(\partial_{t}(A \bar{u})+\partial_{x}\left(\frac{Q^{2}}{A}\right)\right)+\partial_{x} I_{1}(x, A)+\mu_{2} A Q[A, d, \sigma]\left(\frac{Q}{A}\right)=I_{2}(x, A)+O\left(\mu_{2}^{2}\right)
\end{array}\right.
$$

where $Q=A \bar{u}$ is the discharge, $I_{d}$ is the identity operator, $\mathcal{L}$ is a linear operator

$$
\begin{aligned}
\mathcal{L}[A, d, \sigma](u)= & \frac{1}{A}\left[\partial_{x}(\overline{\mathcal{T}}[A, d, \sigma](u, \sigma))-\overline{\mathcal{T}}[A, d, \sigma]\left(u, \partial_{x} \sigma\right)\right] \\
& +\frac{1}{A} \sigma(x) d^{\prime}(x) \mathcal{T}[A, d, \sigma, z=d(x)](u)
\end{aligned}
$$

and $Q$ is a quadratic operator

$$
\begin{aligned}
Q[A, d, \sigma](u)= & \frac{1}{A}\left[\partial_{x}(\overline{\mathcal{G}}[A, d, \sigma](u, \sigma))-\overline{\mathcal{G}}[A, d, \sigma]\left(u, \partial_{x} \sigma\right)\right] \\
& +\frac{1}{A} \sigma(x) d^{\prime}(x) \mathcal{G}[A, d, \sigma, z=d(x)](u)
\end{aligned}
$$

with $\mathcal{T}, \mathcal{G}$ are given by

$$
\mathcal{T}[A, d, \sigma, z](u)=\partial_{x}(u) \int_{z}^{\eta} \frac{S(x, s)}{\sigma(x)} d s+u \int_{z}^{\eta} \frac{1}{\sigma(x)} \partial_{x} S(x, s) d s,
$$

and

$$
\mathcal{G}[A, d, \sigma, z](u)=\int_{z}^{\eta} 2\left(\partial_{x} u\right)^{2} \frac{S(x, s)}{\sigma(x)}+\frac{u^{2}}{\sigma(x)}\left(\frac{\partial_{x} S(x, s) \partial_{x} \sigma(x)}{\sigma(x)}-\partial_{x} \partial_{x} S(x, s)\right)+\partial_{x}\left(\frac{u^{2}}{2}\right) \frac{S(x, s) \partial_{x} \sigma(x)}{\sigma(x)^{2}} d s
$$

with

$$
\bar{X}[A, d, \sigma](u, \psi)=\int_{d(x)}^{\eta} \psi \mathcal{X}[A, d, \sigma, z](u) d z
$$

In particular, one can explicitly compute those operators: 
- if $\sigma \in \mathbb{R}_{*}^{+}$and $d \in \mathbb{R}$ are constant then we recover the standard one-dimensional SGN equations (see for instance $[14,15,16])$ over flat bottom with

$$
\mathcal{L}[A, d, \sigma](u)=\mathcal{L}_{0}[A, \sigma](u)=\frac{1}{\sigma h} \partial_{x}\left(\frac{\sigma h^{3}}{3} \partial_{x} u\right)
$$

and

$$
Q[A, d, \sigma](u)=Q_{0}[A, \sigma](u)=\frac{1}{\sigma h} \partial_{x}\left(\frac{2}{3} \sigma h^{3}\left(\partial_{x} u\right)^{2}\right) .
$$

- if $\sigma \in \mathbb{R}_{*}^{+}$is constant and $d=d(x)$ then we recover the standard one-dimensional SGN equations (see for instance $[14,15,16])$ over uneven bottom with

$$
\mathcal{L}[A, d, \sigma](u)=\mathcal{L}_{1}[A, d, \sigma](u)=\mathcal{L}_{0}[A, \sigma](u)-\frac{1}{\sigma h} \partial_{x}\left(\frac{\sigma h^{2}}{2} u d^{\prime}(x)\right)+\frac{h}{2} \partial_{x} u d^{\prime}(x)-u\left(d^{\prime}(x)\right)^{2}
$$

and

$$
Q[A, d, \sigma](u)=Q_{1}[A, d](u)=Q_{0}[A, \sigma](u)+\frac{1}{\sigma h} \partial_{x}\left(\sigma \frac{h^{2}}{2} u^{2} d^{\prime \prime}(x)\right)+h\left(\partial_{x} u\right)^{2} d^{\prime}(x)+u^{2} d^{\prime}(x) d^{\prime \prime}(x) .
$$

- if $\sigma=\sigma(x)$ and $d=d(x)$ then we get the generalised one-dimensional SGN equations for non-uniform rectangular channel over uneven bottom with

$$
\mathcal{L}[A, d, \sigma](u)=\mathcal{L}_{1}[A, d, \sigma](u)+\frac{1}{\sigma h} \partial_{x}\left(\sigma^{\prime}(x) \frac{h^{3}}{3} u\right)-\frac{\sigma^{\prime}(x)}{\sigma}\left(\partial_{x} u \frac{h^{2}}{3}+u \frac{h^{2}}{3} \frac{\sigma^{\prime}(x)}{\sigma}-u \frac{h}{2} d^{\prime}(x)\right)
$$

and

$$
\begin{aligned}
Q[A, d, \sigma](u)= & Q_{1}[A, d, \sigma](u)+\frac{1}{\sigma h} \partial_{x}\left(\left(\sigma^{\prime}(x)\right)^{2} \frac{u^{2}}{\sigma} \frac{h^{3}}{3}\right)+\frac{1}{\sigma h} \partial_{x}\left(d^{\prime}(x) \sigma^{\prime}(x) u^{2} \frac{h^{2}}{2}\right) \\
& -\frac{1}{\sigma h} \partial_{x}\left(\sigma^{\prime}(x) u^{2} \frac{h^{3}}{3}\right)+\partial_{x}\left(\partial_{x}\left(\frac{u^{2}}{2}\right) \sigma^{\prime}(x) \frac{h^{3}}{3}\right)-\frac{1}{\sigma h} \sigma^{\prime}(x) \mathcal{R}[A, d, \sigma](u)
\end{aligned}
$$

with

$$
\begin{aligned}
\mathcal{R}[A, d, \sigma](u)= & \left(\partial_{x} u\right)^{2} \frac{h^{3}}{3}+u^{2}\left(\frac{\sigma^{\prime}(x)}{\sigma}\right)^{2} \frac{h^{3}}{3}+u^{2}\left(\frac{\sigma^{\prime}(x)}{\sigma}\right) d^{\prime}(x) \frac{h^{2}}{2}-u^{2}\left(\frac{\sigma^{\prime \prime}(x)}{\sigma}\right)^{2} \frac{h^{3}}{3}+u^{2} d^{\prime \prime}(x) \frac{h^{2}}{2} \\
& +\partial_{x}\left(\frac{u^{2}}{2}\right) \frac{\sigma^{\prime}(x)}{\sigma} \frac{h^{3}}{3}-u^{2} d^{\prime}(x) \frac{\sigma^{\prime}(x)}{\sigma} \frac{h^{2}}{2}-u^{2} \sigma^{\prime}(x)\left(d^{\prime}(x)\right)^{2} h+u^{2} \sigma^{\prime \prime}(x) d^{\prime}(x) \frac{h^{2}}{2} \\
& -\partial_{x}\left(\frac{u^{2}}{2}\right) \sigma^{\prime}(x) d^{\prime}(x) \frac{h^{2}}{2}
\end{aligned}
$$

It is known that third order derivatives involved in the initial model (23) may create high frequencies instabilities, but the presence of the $\left(I_{d}-\mu_{2} \mathbb{L}[A, d, \sigma]\right)^{-1}$ in the second equation of (26) stabilises the equations with respect to these perturbations. Therefore, in the following, we construct a numerical scheme for Eqs. (26) instead of Eqs. (23).

\subsection{Numerical method}

This section is devoted to the numerical method to solve the reformulated dispersive model (26). It is rather natural to split the hyperbolic part to the dispersive one as done by several authors (see for instance [5, 4, 3]).

Let $N \in \mathbb{N}^{*}$. Let us consider the following uniform mesh on $\left[0, L_{c}\right]$. Cells are denoted for every $i \in[0, N+1]$, by $m_{i}=\left(x_{i-1 / 2}, x_{i+1 / 2}\right)$ with $x_{i}=\frac{x_{i-1 / 2}+x_{i+1 / 2}}{2}$ the cell center and $\delta x=x_{i+1 / 2}-x_{i-1 / 2}$ the space mesh. The interfaces $x_{1 / 2}=0$ and $x=x_{N+1 / 2}$ denote the upstream and the downstream ends. We also consider a time discretisation $t_{n}$ defined by $t_{n+1}=t_{n}+\delta t_{n}$ where the time step $\delta t_{n}$ is computed through a CFL condition related to the hyperbolic part. 
Let us first highlight that the still water steady state for Eqs. (26) is independent of $\mu_{2}$. Indeed, one has $\forall \mu_{2}>0$, the still water steady state equation reads

$$
u=0, \frac{A}{\sigma}+d=h_{0}
$$

for some positive $h_{0}$. As a consequence, the construction of a well-balanced scheme can be easily achieved considering only the hyperbolic part of Eqs. (26), for instance, by the use of the hydrostatic reconstruction (see for instance [1]).

Let us define $d_{i+1 / 2}=\max \left(d_{i}, d_{i+1}\right)$ where $d_{i}=\frac{1}{\delta x} \int_{m_{i}} d(x) d x, \sigma_{i+1 / 2}=\max \left(\sigma_{i}, \sigma_{i+1}\right)$ where $\sigma_{i}=$ $\frac{1}{\delta x} \int_{m_{i}} \sigma(x) d x$ and let us define the reconstructed states

$$
A_{i+1 / 2}^{-}=\sigma_{i+1 / 2}\left(\frac{A_{i}}{\sigma_{i}}+d_{i}-d_{i+1 / 2}\right), A_{i+1 / 2}^{+}=\sigma_{i+1 / 2}\left(\frac{A_{i+1}}{\sigma_{i+1}}+d_{i+1}-d_{i+1 / 2}\right)
$$

with

$$
U_{i+1 / 2}^{-}=\left(A_{i+1 / 2}^{-}, A_{i+1 / 2}^{-} u_{i}\right), U_{i+1 / 2}^{+}=\left(A_{i+1 / 2}^{+}, A_{i+1 / 2}^{+} u_{i+1}\right)
$$

where $U_{i}=\left(A_{i}, A_{i} u_{i}\right)^{T} \approx \frac{1}{\delta x} \int_{m_{i}}(A, A u)^{T} d x$.

Let us introduce the flux

$$
F_{1}(U)=Q, F_{2}(U)=Q^{2} / A \text { and } F_{3}(x, U)=I_{1}(x, A)+\mu_{2} \overline{\mathcal{G}}[A, d, \sigma](u, \sigma)
$$

and

$$
\subseteq(x, U)=I_{2}+\mu_{2} \overline{\mathcal{G}}[A, d, \sigma]\left(u, \partial_{x} \sigma\right)-\mu_{2} \sigma(x) d^{\prime}(x) \mathcal{G}[A, d, \sigma, z=d(x)](u) .
$$

Then, one can write System (26) as follows:

$$
\begin{array}{ll}
\partial_{t} A+\partial_{x} F_{1}(U) & =0 \\
\left(I_{d}-\mu_{2} \mathbb{L}[A, d, \sigma]\right)\left(\partial_{t} Q+\partial_{x} F_{2}(U)\right)+\partial_{x} F_{3}(x, U)-\mathfrak{S}(x, U) & =0
\end{array}
$$

With these settings, we define the following numerical scheme:

$$
\begin{aligned}
& A_{i}^{n+1}=A_{i}^{n}-\frac{\delta t_{n}}{\delta x}\left(\mathcal{F}_{1}\left(U_{i+1 / 2}^{-, n}, U_{i+1 / 2}^{+, n}\right)-\mathcal{F}_{1}\left(U_{i-1 / 2}^{-, n}, U_{i-1 / 2}^{+, n}\right)\right) \\
& Q_{i}^{*}=Q_{i}^{n}-\frac{\delta t_{n}}{\delta x}\left(\mathcal{F}_{2}\left(U_{i+1 / 2}^{-, n}, U_{i+1 / 2}^{+, n}\right)-\mathcal{F}_{2}\left(U_{i-1 / 2}^{-, n}, U_{i-1 / 2}^{+, n}\right)\right) \\
& Q_{i}^{n+1}=Q_{i}^{*}-\frac{\delta t_{n}}{\delta x}\left(Y^{n}\right)_{i}
\end{aligned}
$$

where

$$
\mathcal{A}^{n} Y^{n}=\left(\mathcal{F}_{3}\left(x_{i+1 / 2}, U_{i+1 / 2}^{-, n}, U_{i+1 / 2}^{+, n}\right)-\mathcal{F}_{3}\left(x_{i-1 / 2}, U_{i-1 / 2}^{-, n}, U_{i-1 / 2}^{+, n}\right)+\mu_{2} \mathcal{N}_{i}^{n}\right)_{1 \leq i \leq N} .
$$

The matrix $\mathcal{A}^{n}$ is the cell-centered approximation of the linear operator $\left(I_{d}-\mu_{2} \mathbb{L}[A, d, \sigma]\right)$ and $\mathcal{N}_{i}^{n}$ is the cell-centered approximation of $-\overline{\mathcal{G}}[A, d, \sigma]\left(u, \partial_{x} \sigma\right)+\sigma(x) d^{\prime}(x) \mathcal{G}[A, d, \sigma, z=d(x)](u)$.

The numerical fluxes are defined by

$$
\begin{aligned}
& \mathcal{F}_{1}\left(U_{i+1 / 2}^{-, n}, U_{i+1 / 2}^{+, n}\right) \quad=\frac{F_{1}\left(U_{i+1 / 2}^{-, n}\right)+F_{1}\left(U_{i+1 / 2}^{+, n}\right)}{2}-s_{i+1 / 2}^{n}\left(A_{i+1 / 2}^{+, n}-A_{i+1 / 2}^{-, n}\right) \\
& \mathcal{F}_{2}\left(U_{i+1 / 2}^{-, n}, U_{i+1 / 2}^{+, n}\right) \\
& \mathcal{F}_{3}\left(x_{i+1 / 2}, U_{i+1 / 2}^{-, n}, U_{i+1 / 2}^{+, n}\right)=\frac{F_{2}\left(U_{i+1 / 2}^{-, n}\right)+F_{2}\left(U_{i+1 / 2}^{+, n}\right)}{2}-s_{i+1 / 2}^{n}\left(Q_{i+1 / 2}^{+, n}-Q_{i+1 / 2}^{-, n}\right) \\
& \mathcal{F}_{3}\left(x_{i+1 / 2}, U_{i+1 / 2}^{-, n}, U_{i+1 / 2}^{+, n}\right)=\frac{F_{3}\left(x_{i+1 / 2}, U_{i+1 / 2}^{-, n}\right)+F_{3}\left(x_{i+1 / 2}, U_{i+1 / 2}^{+, n}\right)}{2}+\left(\frac{A_{i}^{n} 2}{\left.2 \sigma_{i} F_{r+1 / 2}, U_{i+1 / 2}^{+,}\right)}-\frac{A_{i+1 / 2}^{+, n}{ }^{2}}{2 \sigma_{i+1 / 2} F_{r}^{2}}\right) \\
&
\end{aligned}
$$


such that whenever $\mu_{2}=0$, we recover the classical numerical scheme ${ }^{1}$ for the hyperbolic part

$$
U_{i}^{n+1}=U_{i}^{n}-\frac{\delta t_{n}}{\delta x}\left(\mathcal{F}\left(x_{i+1 / 2}, U_{i+1 / 2}^{-, n}, U_{i+1 / 2}^{+, n}\right)-\mathcal{F}\left(x_{i-1 / 2}, U_{i-1 / 2}^{-, n}, U_{i-1 / 2}^{+, n}\right)\right)
$$

with $\mathcal{F}(x, U, V)=\left(\mathcal{F}_{1}(U, V), \mathcal{F}_{2}(U, V)+\mathcal{F}_{3}(x, U, V)\right)$. In these expressions,

$$
s_{i+1 / 2}=\max _{j=1,2}\left|\lambda_{j}\left(x_{i+1 / 2}, U_{i+1 / 2}^{-, n}\right)\right|,\left|\lambda_{j}\left(x_{i+1 / 2}, U_{i+1 / 2}^{+, n}\right)\right|
$$

where $\lambda_{j}(x, U)=Q / A+(-1)^{j} \sqrt{\frac{A}{\sigma(x) F_{r}^{2}}}, j=1,2$ are the eigenvalues of the Jacobian matrix of $\left(F_{1}, F_{2}+F_{3}\right)^{T}$.

The numerical scheme is consistent and stable under the CFL condition

$$
\max _{1 \leq i \leq N}\left(\left|\lambda_{1}\left(x_{i}, U_{i}^{n}\right)\right|,\left|\lambda_{2}\left(x_{i}, U_{i}^{n}\right)\right|\right) \frac{\delta t_{n}}{\delta x} \leq 1 .
$$

\subsection{Propagation of a solitary wave}

In this section, we test the accuracy of the method and we show numerically the influence of the section variation in the case of the propagation of a solitary wave. For this purpose, we consider the exact solitary wave solutions of the Green-Naghdi equations in the one-dimensional setting over a flat bottom (see [15]), given in variables with dimensions, by

$$
\eta(t, x)=a \operatorname{sech}^{2}(k(x-c t)), \quad u(t, x)=c\left(\frac{\eta(t, x)}{\eta(t, x)+z_{0}}\right) \text { with } k=\frac{\sqrt{3 a}}{2 z_{0} \sqrt{z_{0}+a}} \text { and } c=\sqrt{g\left(z_{0}+a\right)}
$$

where $z_{0}$ is the depth of the fluid and $a$ is the relative amplitude.

\section{Accuracy}

The propagation of the solitary wave (27) is initially centered at $x_{0}=10 \mathrm{~m}$ with a relative amplitude $a=0.2$ $\mathrm{m}$ over a constant water depth $z_{0}=2 \mathrm{~m}$. The computational domain is $L_{c}=100 \mathrm{~m}$ and it is discretized with $N$ cells. The single solitary wave propagates from left to right. In this test, since the solitary wave is initially far from boundaries, the boundary conditions do not affect the computation, thus we choose to impose free boundary conditions at the downstream and upstream ends. The exact solution is computed in a channel of width $\sigma=1$.

In what follows, we quantify the numerical accuracy of our numerical scheme by computing the numerical solution for this particular test case for an increasing number of cells $N$ over a duration $T=20 \mathrm{~s}$. Starting with $N=100$ number of cells, we successively multiply the number of cells by two. For all $n$, we compare, in Fig. $2, M^{n}:=\max _{0 \leq i \leq N+2}\left(h_{i}^{n}\right)$ of our numerical solution provided by Eqs. (26) with the exact one $M\left(t_{n}\right):=\max h\left(t_{n}, x\right)_{x \in\left[0, L_{c}\right]}=2.2$ given by (27). One can easily remark that the first order discretisation is not accurate for long time simulation due to the numerical dissipation. However, to limit the numerical dissipation of the first order numerical scheme, one can either limit the simulation time or consider a very large number of cells. However, it is better to increase the order of the numerical scheme but this is left to future work. Therefore, in what follows, we consider a shorter simulation time and a large number of cells, just to illustrate the influence of the variation of the channel.

\footnotetext{
${ }^{1}$ For the sake of simplicity and clarity, we have presented the finite volume method using the Rusanov solver but the method is not limited to this one.
} 
Fig. $2 M^{n}:=\max _{0 \leq i \leq N+2}\left(h_{i}^{n}\right)$

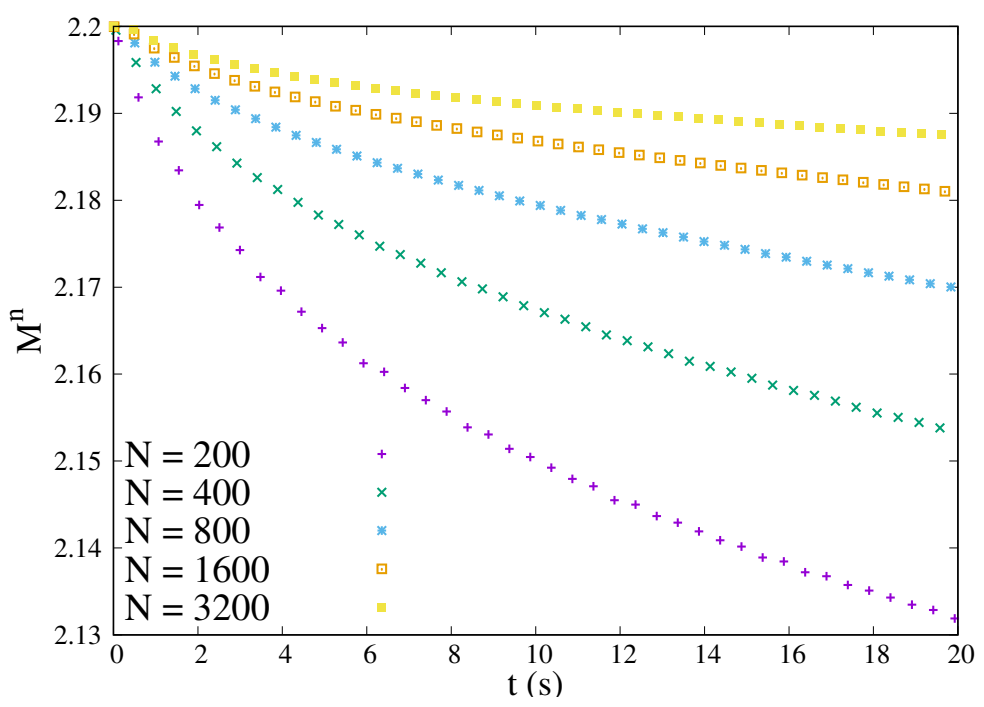

\section{Influence of the section variation:}

We consider again the propagation of a solitary wave initially centered at $x_{0}=10 \mathrm{~m}$ of relative amplitude $a=0.2 \mathrm{~m}$, over a constant water depth $z_{0}=2 \mathrm{~m}$ onto a computational domain of $L_{c}=50 \mathrm{~m}$ and discretized with $N=5000$ cells. The final simulation time is $T=8 \mathrm{~s}$. Initially starting with $(\eta(0, x), u(0, x))$ (see Eqs. (27)), we compute the numerical simulation for the channels defined by

$$
\left.\sigma(x ; \varepsilon)=\beta(x ; \varepsilon)-\alpha(x ; \varepsilon) \text { with } \beta=\frac{1}{2}-\frac{\varepsilon}{2} \exp \left(-\varepsilon^{2}(x-L / 2)^{2}\right)\right) \text { and } \alpha=-\beta
$$

with $\epsilon=0, \epsilon=0.1, \epsilon=0.2, \epsilon=0.3$ and $\epsilon=0.4$. The obtained results are presented in Fig. 3. In Fig.4(a), for each geometry, we show the evolution of the maximum of the water level $M^{n}:=\max _{0 \leq i \leq N+2}\left(h_{i}^{n}\right)$. As expected, since the first part for $x \leq 25$ is linearly converging, the water level increases while for $x>25$, the channel is linearly diverging and therefore, the amplitude of the water level decreases. Moreover, in all numerical simulations, the mass is conserved. Indeed, for each value of $\varepsilon$, we have displayed in Fig. 4(b), the ratio of $\frac{m^{n}}{m^{0}}$ where $m^{n}:=\frac{1}{N+2} \sum_{i=0}^{N+1} A_{i}^{n}$ is the mass of water at time $t_{n}$. The ratio $\frac{m^{n}}{m^{0}}$ is almost equal to 1 , up to the order of accuracy of the numerical scheme. 
Fig. 3 Influence of $\sigma$

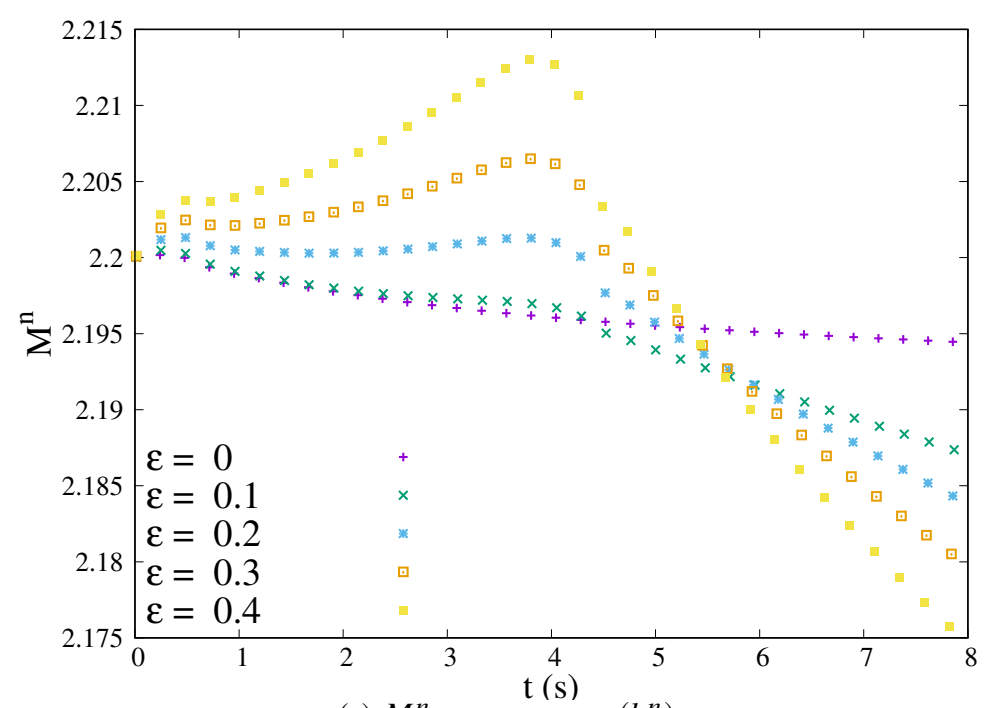

(a) $M^{n}:=\max _{x \in\left[0, L_{c}\right]}\left(h_{i}^{n}\right)$

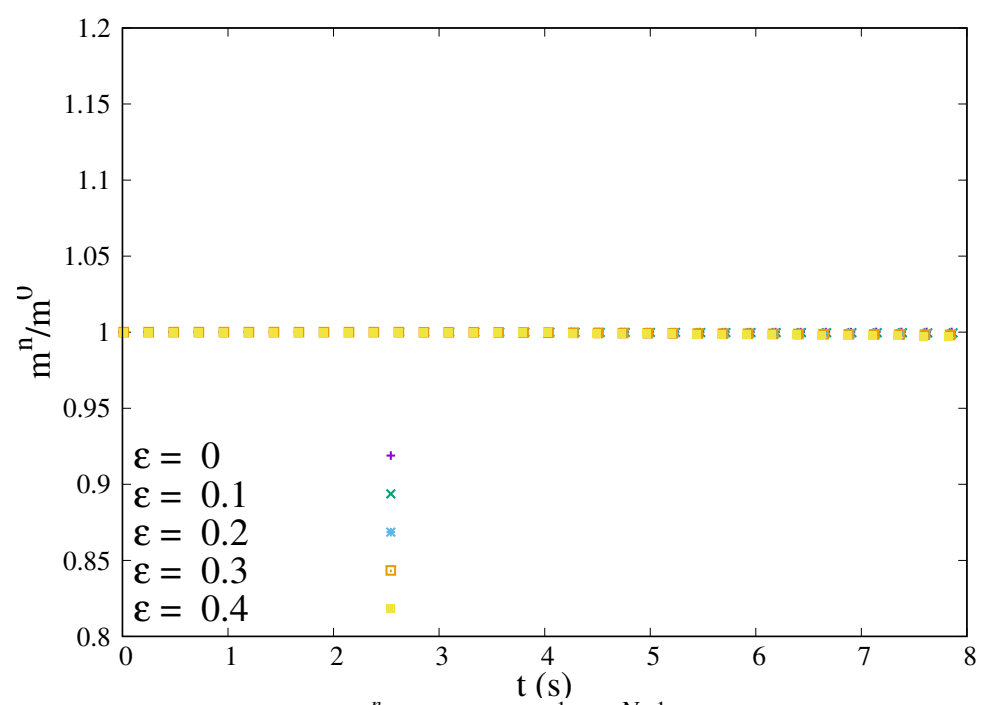

(b) $\frac{m^{n}}{m^{0}}$ with $m^{n}=\frac{1}{N+2} \sum_{i=0}^{N+1} A_{i}^{n}$

In what follows, we quantify the numerical accuracy of our numerical scheme. Starting with $N=100$ number of cells, we successively multiply the number of cells by two. The errors on the water surface deformation are presented in Table 1 and in Table 2. These errors are computed at $t=8 \mathrm{~s}$ using the $L^{2}$ :

$$
\left\|\eta_{\text {num }}-\eta_{\text {ref }}\right\|_{2}=\sqrt{\delta x \sum_{i}\left|\eta_{\text {num }_{i}}(t=8)-\eta_{\text {ref }}\left(t=8, x_{i}\right)\right|}
$$

and the $L^{\infty}$ norms where $\eta_{\text {ref }}$ is the exact solution in the case $\varepsilon=0$ and is a reference one computed with 10000 cells for $\varepsilon=0.4$. Since the results are almost the same whatever $\varepsilon$ is, we have decided to present only the results for $\varepsilon=0$ and $\varepsilon=0.4$ in Table 1 and in Table 2 .

As expected, the obtain numerical order is slow because of the numerical dissipation of the solitary wave (as already pointed out in several works, see for instance [3] for which we obtain almost the same order 
of convergence in the case of uniform section). Moreover, as expected, cross-sectional variations have no influence on the convergence rate. Let us just emphasise that the convergence rates are slightly better in the case of non-uniform section because we are comparing our results to a reference solution and not to the exact one.

Table 1 Convergence rate of the $L^{2}$ error for $\varepsilon=0$. The order is computed through first order interpolation polynomial

\begin{tabular}{lll}
\hline$N$ & $\left\|\eta_{\text {num }}-\eta_{\text {exact }}\right\|_{2}$ & $\left\|\eta_{\text {num }}-\eta_{\text {exact }}\right\|_{\infty}$ \\
\hline 100 & 0.0789 & 0.0449 \\
200 & 0.0497 & 0.0288 \\
400 & 0.0304 & 0.0180 \\
800 & 0.0198 & 0.0116 \\
1600 & 0.0153 & 0.0081 \\
3200 & 0.0138 & 0.0062 \\
Order & 0.53 & 0.58 \\
\hline
\end{tabular}

Table 2 Convergence rate of the $L^{2}$ error for $\varepsilon=0.4$. The reference solution is computed with 10000 cells. The order is computed through first order interpolation polynomial

\begin{tabular}{lll}
\hline$N$ & $\left\|\eta_{\text {num }}-\eta_{\text {ref }}\right\|_{2}$ & $\left\|\eta_{\text {num }}-\eta_{\text {ref }}\right\|_{\infty}$ \\
\hline 100 & 0.05212 & 0.02533 \\
200 & 0.02096 & 0.01082 \\
400 & 0.01079 & 0.00554 \\
800 & 0.00748 & 0.00503 \\
1600 & 0.00635 & 0.00412 \\
3200 & 0.00505 & 0.00300 \\
Order & 0.64 & 0.56 \\
\hline
\end{tabular}

\section{Conclusions and perspectives}

We have presented the derivation of a new dispersive model for open channel with non-uniform rectangular section. This model generalises the usual Serre-Green-Naghdi equation. We have presented its numerical finite volume approximation for which we have proposed two simple test cases. In a forthcoming paper, we will focus on the case of arbitrary channel section and we will propose a high order numerical scheme.

\section{Acknowledgment}

The authors thank the referees for their valuable remarks which led to a substantial improvement of the first version of this paper. The second author wishes to grateful Dr Griggio for her help throughout this work and would like to say sincerely "Sağol".

\section{References}

1. Audusse, E., Bouchut, F., Bristeau, M.O., Klein, R., Perthame, B.: A fast and stable well-balanced scheme with hydrostatic reconstruction for shallow water flows. SIAM J. Sci. Comput. 25, 2050-2065 (2004) 
2. Bourdarias, C., Ersoy, M., Gerbi, S.: A mathematical model for unsteady mixed flows in closed water pipes. Sci. China. Math 55, 221-244 (2012)

3. Bourdarias, C., Gerbi, S., Lteif, R.: A numerical scheme for an improved green-naghdi model in the camassa-holm regime for the propagation of internal waves. Comput. Fluids 156, 283-304 (2017)

4. Chazel, F., Lannes, D., Marche, F.: Numerical simulation of strongly nonlinear and dispersive waves using a green-naghdi model. J. Sci. Comput. 48, 105-116 (2011)

5. Cienfuegos, R., Barthélemy, E., Bonneton, P.: A fourth-order compact finite volume scheme for fully nonlinear and weakly dispersive boussinesq-type equations. part ii: boundary conditions and validation. Int. J. Numer. Methods. Fluids. 53, $1423-1455$ (2007)

6. Debyaoui, M.A., Ersoy, M.: Generalised Serre-Green-Naghdi equations for open channel and for natural river hydraulics (2020). URL https://hal.archives-ouvertes.fr/hal-02444355. Working paper or preprint

7. Decoene, A., Bonaventura, L., Miglio, E., Saleri, F.: Asymptotic derivation of the section-averaged shallow water equations for natural river hydraulics. Math. Models Methods Appl. Sci. 19, 387-417 (2009)

8. Ersoy, M.: Dimension reduction for incompressible pipe and open channel flow including friction. In: Conference Applications of Mathematics 2015, in honor of the 90th birthday of Ivo Babuska and 85th birthday of Milan Práger and Emil Vitásek, pp. 17-33. J. Brandts and S. Korotov and M. Krizek and K. Segeth and J. Sistek and T. Vejchodsky, Prague, France (2015). URL https://hal.archives-ouvertes.fr/hal-00908961

9. Fedotova, Z.I., Khakimzyanov, G.S., Dutykh, D.: Energy equation for certain approximate models of long-wave hydrodynamics. Russ. J. Numer. Anal. Math. Model. 29, 167-178 (2014)

10. Gerbeau, J.F., Perthame, B.: Derivation of viscous Saint-Venant system for laminar shallow water; numerical validation. Discrete. Continuous. Dyn. Syst. Ser. B 1, 89-102 (2001)

11. Gouta, N., Maurel, F.: A finite volume solver for $1 \mathrm{~d}$ shallow-water equations applied to an actual river. Int. J. Numer. Methods. Fluids. 38, 1-19 (2002)

12. Green, A.E., Naghdi, P.M.: A derivation of equations for wave propagation in water of variable depth. J. Fluid Mech. 78, 237-246 (1976)

13. Lannes, D.: The Water Waves Problem: Mathematical Analysis and Asymptotics, vol. 188. Am. Math. Soc. (2013)

14. Lannes, D., Bonneton, P.: Derivation of asymptotic two-dimensional time-dependent equations for surface water wave propagation. Phys. Fluids 21, 016601 (2009)

15. Lannes, D., Marche, F.: A new class of fully nonlinear and weakly dispersive green-naghdi models for efficient $2 d$ simulations. J. Comput. Phys. 282, 238-268 (2015)

16. Lannes, D., Marche, F.: Nonlinear wave-current interactions in shallow water. stud. appl. math. 136, $382-423$ (2016)

17. Peregrine, D.: Calculations of the development of an undular bore. J. Fluid Mech. 25, 321-330 (1966)

18. de Saint-Venant, A.J.C.B.: Théorie du mouvement non-permanent des eaux, avec application aux crues des rivières et à l'introduction des marées dans leur lit. C. R. Acad. Sci 73, 147-154 (1871)

19. Seabra-Santos, F.J., Renouard, D.P., Temperville, A.M.: Numerical and experimental study of the transformation of a solitary wave over a shelf or isolated obstacle. J. Fluid Mech. 176, 117-134 (1987) 